\title{
Personalized Medicine: Challenging Pharmaceutical and Diagnostic Company Business Models
}

\author{
Joseph Ferrara
}

\begin{abstract}
The phrase "Personalized Medicine" has been alternately broadly and narrowly defined. Under its broadest definition, clinicians have been have been providing personalized medicine for as long as there has been medical practice - physicians employ diagnostic tools, whether in vitro tests, imaging techniques, or symptomology to determine a particular condition, and then go on to prescribe the appropriate treatment, drug or otherwise.

However, it is pharmacogenomics -- the ability to test for variations in genes and their expression through molecular diagnostics and then to treat with targeted drugs -- that shapes a narrower, and more potent, definition of personalized medicine. For patients and physicians, personalized medicine offers apparently clear clinical advantages -- a biomarker points directly to the most appropriate therapeutic intervention. This in turn leads to more predictable outcomes, enhanced efficacy through the identification of drug responders, and a potential reduction in adverse drug reactions. This would be a sea change for at least some of medical practice, where certain drugs may now be prescribed on something of a trial and error basis and the therapy effective for only a portion of the treated patients.

While personalized medicine offers the potential to change well-established practices for physicians and patients, the concept presents a direct challenge to the business models of two other health care stakeholders essential to the realization of personalized medicinepharmaceutical and diagnostics companies. At the core of the challenge is a central question- how will a personalized medicine paradigm change these companies' innovation and commercialization approaches? This question can be aimed at nearly every aspect of these stakeholders' current strategies- how
\end{abstract}

clinical trials are conducted, the depth and breadth of the clinical evidence developed, and, perhaps most importantly, how value is created and captured.

\section{PHARMACEUTICAL COMPANIES- AN OPPORTUNISTIC APPROACH TO PHARMACOGENOMICS}

Pharmacogenomics is a growing component of drug development, but drug companies' desire to sell blockbuster drugs (drugs with annual sales of one billion dollars or more) will not disappear anytime soon. The blockbuster model has dominated $R \& D$ and commercialization planning for large pharmaceutical companies since the 1980s. In 2002, biopharmaceutical companies marketed 40 blockbuster drugs with total sales of nearly $\$ 90$ billion. However, studies have shown that many of these drugs may be efficacious in only slim majority of the prescribed population. The model is so entrenched, that physicians often prescribe these drugs on a trial and error basis. And, of course, these drugs demand large, expensive trials. However, drug companies' substantial investment in $R \& D$ to find more of these drugs has yet to yield a proportional increase in new approved drugs.

With such a low yielding research investment environment, pharmacogenomics offers the potential at least, to make up some of the shortfall. In 2005, the FDA created guidance for drug developers to link biomarkers to therapeutics in the clinical development process. The FDA views pharmacogenomics as a key tool for optimizing both the development and clinical utility of drugs.

Perhaps not surprisingly, the majority of diagnostic/pharmaceutical (Dx/Rx) combinations are in oncology. Besides being a major magnet for 
pharmaceutical R\&D investment, oncology presents a tailor-made target for pharmacogenomics- cancer is a complex, multi-factorial disease. It is also a clinical area many stakeholders, not just industry, view as an optimal target for $\mathrm{Dx} / \mathrm{Rx}$ combinations- the disease is life threatening, the drugs often have brutal side effects, and these treatments are often very expensive. Identifying tests that can help target these drugs to only those patients that benefit most has great value in this clinical area.

With all of these potential benefits to pharmacogenomics, some drug companies have responded by increasing their investment in the $\mathrm{Dx} / \mathrm{Rx}$ approach. The number of INDs/NDAs using pharmacogenomic biomarkers has increased from less than 20 filings between 1995 and 2000 to nearly 100 filings in the 2002-2003 period alone. Many of the filings have been in oncology, and based on an analysis of the current cancer pipeline and estimated clinical trial success rates, there may be as many as 35 new targeted cancer therapies on the US market by 2012. And while all of these drugs could be candidates for a $\mathrm{Dx} / \mathrm{Rx}$ combination, just how many of these drugs will launch with a companion diagnostic test remains to be seen because of the unpredictability of these combinations in clinical development and practice.

Drug companies have had mixed results in effectively incorporating diagnostic biomarkers into clinical development programs and then launching the combination. The most well known Dx/Rx combination is the HercepTest $\AA /$ trastuzumab (Herceptin $®)$ combination from Dako and Genentech/Roche for the Her2-positive subset of breast cancer patients. However, less successful In terms of Dx / Rx pairings have been the cases of cetuximab (Erbitux®) and gefitinib (Iressa $\left.{ }^{\circledR}\right)$. As has been widely documented, both cases have demonstrated that it can be difficult to predict which drugs will benefit from companion biomarkers, with some studies showing that cefitinib (launched with a companion EGFR test) may have a benefit in a portion of EGFR-negative patients and gefitinib (launched without a companion test) may indeed need a test to identify the subset of optimal responders.

The difficulty in predicting the clinical utility of biomarkers in drug development in tandem with the perceived threat to the blockbuster model means that pharmaceutical companies have embraced pharmacogenomics with varying degrees of enthusiasm and investment. Although several drug companies have publicly committed to developing and launching drugs with attendant diagnostic tests, it is difficult to imagine that these companies will abandon the quest for the billion dollar drug in favor of an rarely proven pharmacogenomic commercial model. Most likely, big drug companies will take a "both-and" approach to drug development and commercialization-continuing to pursue the high revenue potential of a "one-size-fitsmost" product while opportunistically incorporating pharmacogenomics approaches. Success in the latter will much depend on the relationships that pharmaceutical players develop, directly or indirectly, collaborative or adversarial, with the other key player in the tandem- diagnostics companies.

\section{DIAGNOSTICS COMPANIES: PURSUING VALUE-BASED RECOGNITION}

It is the diagnostics companies that have the most to gain in the near term from the realization of personalized medicine. This is largely because in vitro diagnostics are currently so undervalued compared to their drug counterparts in the first place.

For example, in the US, for every ten dollars that Medicare spends on pharmaceuticals, the agency spends roughly $\$ 1.60$ on lab services. And laboratory services influence not only pharmaceutical therapy selection, but other interventions as well-- perhaps as much as $60 \%$ to $70 \%$ of health care expenditures according to some studies.

However, the system that payers use to reimburse for diagnostics has become a limiting factor in the ability for innovative diagnostics to be appropriately valued in the commercial environment. Currently, payers reimburse for laboratory services using what is largely a cost-based system, not a value-based system.

Currently, payment amounts for new tests are typically established relative to older tests with similar analytical methodologies. Thus, any new immunoassay will likely be reimbursed at a rate similar to established immunoassays. The same is true in molecular diagnostics. Any new test using established nucleic acid analytical methodologies will be paid the same amounts as older tests using the same methodologies, ignoring the likely difference in reagent expenses between the older and the newer tests. It would be as if all novel injectable drugs were reimbursed at the same amounts as injectables that have been on the market for many years. Such an approach does not allow for a novel payment amount to be applied to a novel test- a payment amount that could be related to the test's perceived clinical value, for example.

To see how this cost-based system has hampered value creation for the diagnostics industry, one needs only to look at the trend in Medicare reimbursement rates over the last twenty years. For example, a test that was paid at $\$ 10$ on average in the mid 1980s is now paid at about perhaps $\$ 8$ after adjusting for inflation. Stakeholders, and not just industry groups, understand the value of innovation in medicine. A decline in payment of $20 \%$ in real dollars over two decades is not 
an environment conducive to the innovation that will be required to drive pharmacogenomics and personalized medicine.

Conceptually, personalized medicine could allow for a reconfiguration of the value of the parts in the Dx/Rx whole. The idea is that payers will begin to look at the diagnostic as an increasingly valuable tool that can help manage the appropriate use of expensive drugs. If a test can be used to avoid even a few months of an expensive prescription for a non-responder, the test marketer can, in theory at least, capture some of this enhanced value.

Diagnostics players understand this, and are attempting to capture novel value for their novel tests in the Dx/Rx paradigm. Logically, developing tests that will distinguish responders from non-responders for expensive drugs or drugs with dangerous side effects is a priority offering both clinical and economic value. To meet this objective, diagnostics companies are deploying two basic business models, each with different approaches to clinical development and market risk.

The longer-term opportunity for these diagnostics companies will be to link test development programs to drug development programs and then launching the approved drug and test as a true tandem from the outset. In this way, compelling clinical evidence for the test and drug will be developed by design, supporting the value of the $\mathrm{Dx} / \mathrm{Rx}$ combination. While a diagnostics company is not taking the same clinical development risk and making the same scale of investment as the drug company in such a model, many of the drugs developed may not be approved, and thus a test could be developed that may never see the market.

A nearer-term opportunity for diagnostics companies is the development of tests that are linked to currently marketed therapies. Currently marketed drugs that could benefit from a test to identify optimal responders are near-term target markets for diagnostics companies. Of course, diagnostics companies that launch tests linked to currently marketed drugs will likely seek reimbursement from payers that reflects the perceived value of their innovation. To do so, these companies will have to take control of the reimbursement process, perhaps working outside of established test payment mechanisms such as payer clinical lab fee schedules, to seek a payment rate not tied to established tests or methodologies. In order to this, diagnostics companies will have to develop adequate clinical data to support positive coverage of the test by payers, and accordingly positive payment.

\section{PERSONALIZED MEDICINE WILL REQUIRE HEALTH CARE STAKEHOLDER COLLABORATION}

The development and delivery of the necessary component parts for personalized medicine solutions will continue to come from numerous industries. This raises an important question-- how will the range of health care stakeholders work together to develop and deploy innovative technologies for personalized medicine?

The circumstances that will allow personalized medicine to flourish are just beginning to take shape. Stakeholders are moving forward with research investment, new models for collaboration, and developing regulatory and policy approaches that can allow for these technologies to emerge. In addition to the FDA guidance on pharmacogenomics in drug development, payers, both government and private, are developing methodologies for translating clinical evidence into policies that will guide insurance payment. And this may be the most challenging issue of all for personalized medicine-developing a policy approach that allows access to these technologies for right patients given the highly targeted nature of personalized medicine. By design, the policy-making environment was formed on a foundation of broadly applicable therapies. For policy makers the question is this- how can policies be developed when many treatments may be decided on a highly varied case-bycase basis? Do we even have the analytical tools and information technology infrastructure to analyze the clinical and economic effectiveness of these highly targeted approaches?

As these efforts continue, it will be essential for diagnostics and pharmaceutical companies, payers, physicians, and patient groups to interact to create an environment that is favorable to both the basic research that is required for innovation of effective personalized medicine solutions, as well as their eventual commercialization.

Joseph V. Ferrara is the Executive Vice President of Boston Healthcare. He has over ten years of experience in life sciences consulting, working with established and emerging biopharmaceutical, medical device, and diagnostics clients. He has extensive experience working with client companies in business development strategy, market opportunity assessment, and reimbursement planning. Mr. Ferrara's particular focus within the consulting practice is in personalized medicine, developing business strategies for biopharmaceutical and in vitro diagnostics companies in this emerging clinical arena. Mr. Ferrara received his graduate degree from Harvard University and holds an undergraduate degree from the University of Cincinnati. 